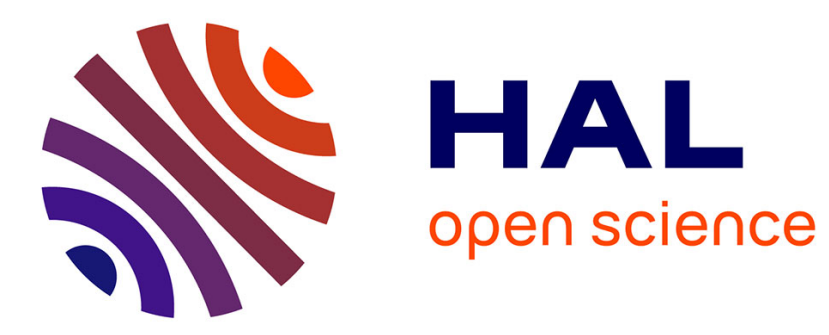

\title{
Engineering Single-Shot All-Optical Switching of Ferromagnetic Materials
}

Junta Igarashi, Quentin Remy, Satoshi Iihama, Grégory Malinowski, Michel

Hehn, Jon Gorchon, Julius Hohlfeld, Shunsuke Fukami, Hideo Ohno, Stéphane Mangin

\section{To cite this version:}

Junta Igarashi, Quentin Remy, Satoshi Iihama, Grégory Malinowski, Michel Hehn, et al.. Engineering Single-Shot All-Optical Switching of Ferromagnetic Materials. Nano Letters, 2020, 10.1021/acs.nanolett.0c03373 . hal-03030501

\section{HAL Id: hal-03030501 \\ https://hal.univ-lorraine.fr/hal-03030501}

Submitted on 1 Dec 2020

HAL is a multi-disciplinary open access archive for the deposit and dissemination of scientific research documents, whether they are published or not. The documents may come from teaching and research institutions in France or abroad, or from public or private research centers.
L'archive ouverte pluridisciplinaire HAL, est destinée au dépôt et à la diffusion de documents scientifiques de niveau recherche, publiés ou non, émanant des établissements d'enseignement et de recherche français ou étrangers, des laboratoires publics ou privés. 


\section{Engineering single-shot all-optical switching of ferromagnetic materials}

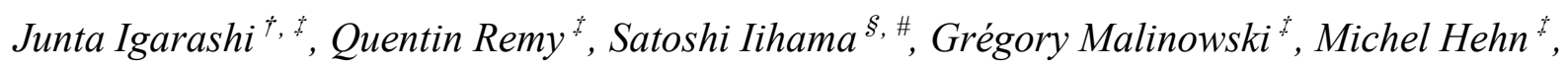

Jon Gorchon ${ }^{*}$, Julius Hohlfeld ${ }^{*}$, Shunsuke Fukami ${ }^{\dagger,}, \|, \perp, \#, \nabla$, Hideo Ohno ${ }^{+,}, \|, \perp, \#, \nabla$, Stéphane Mangin $^{*},+$

${ }^{\dagger}$ Laboratory for Nanoelectronics and Spintronics, Research Institute of Electrical Communication, Tohoku University, 2-1-1 Katahira, Aoba, Sendai 980-8577, Japan.

${ }^{\ddagger}$ Institut Jean Lamour, UMR CNRS 7198, Université de Lorraine, Nancy, France.

${ }^{\S}$ Frontier Research Institute for Interdisciplinary Sciences, Tohoku University, 6-3 Aramaki Aza Aoba, Sendai 980-8578.

"WPI Advanced Institute for Materials Research, Tohoku University, 2-1-1 Katahira, Aoba, Sendai 980-8577, Japan.

${ }^{\perp}$ Center for Science and Innovation in Spintronics, Tohoku University, 2-1-1 Katahira, Aoba, Sendai 980-8577, Japan.

${ }^{\#}$ Center for Spintronics Research Network, Tohoku University, 2-1-1 Katahira, Aoba, Sendai 980-8577, Japan. 
${ }^{\nabla}$ Center for Innovative Integrated Electronic Systems, Tohoku University, 468-1 Aramaki Aza Aoba, Sendai 980-0845, Japan.

\section{KEYWORDS}

All-optical switching, single-shot all-optical switching, spin valve, spin current, ultrafast demagnetization, ultrafast laser 


\section{ABSTRACT}

Since it was recently demonstrated in a spin-valve structure, magnetization reversal of a ferromagnetic layer using a single ultrashort optical pulse has attracted attention for future ultrafast and energy-efficient magnetic storage or memory devices. However, the mechanism and the role of the magnetic properties of the ferromagnet as well as the time scale of the magnetization switching are not understood. Here, we investigate single-shot all-optical magnetization switching in a $\mathrm{GdFeCo} / \mathrm{Cu} /\left[\mathrm{Co}_{x} \mathrm{Ni}_{1-\mathrm{x}} / \mathrm{Pt}\right]$ spin-valve structure. We demonstrate that the threshold fluence for switching both the GdFeCo and the ferromagnetic layer depends on the laser pulse duration and the thickness and the Curie temperature of the ferromagnetic layer. We are able to explain most of the experimental results using a phenomenological model. This work provides a way to engineer ferromagnetic materials for energy efficient single-shot all-optical magnetization switching.
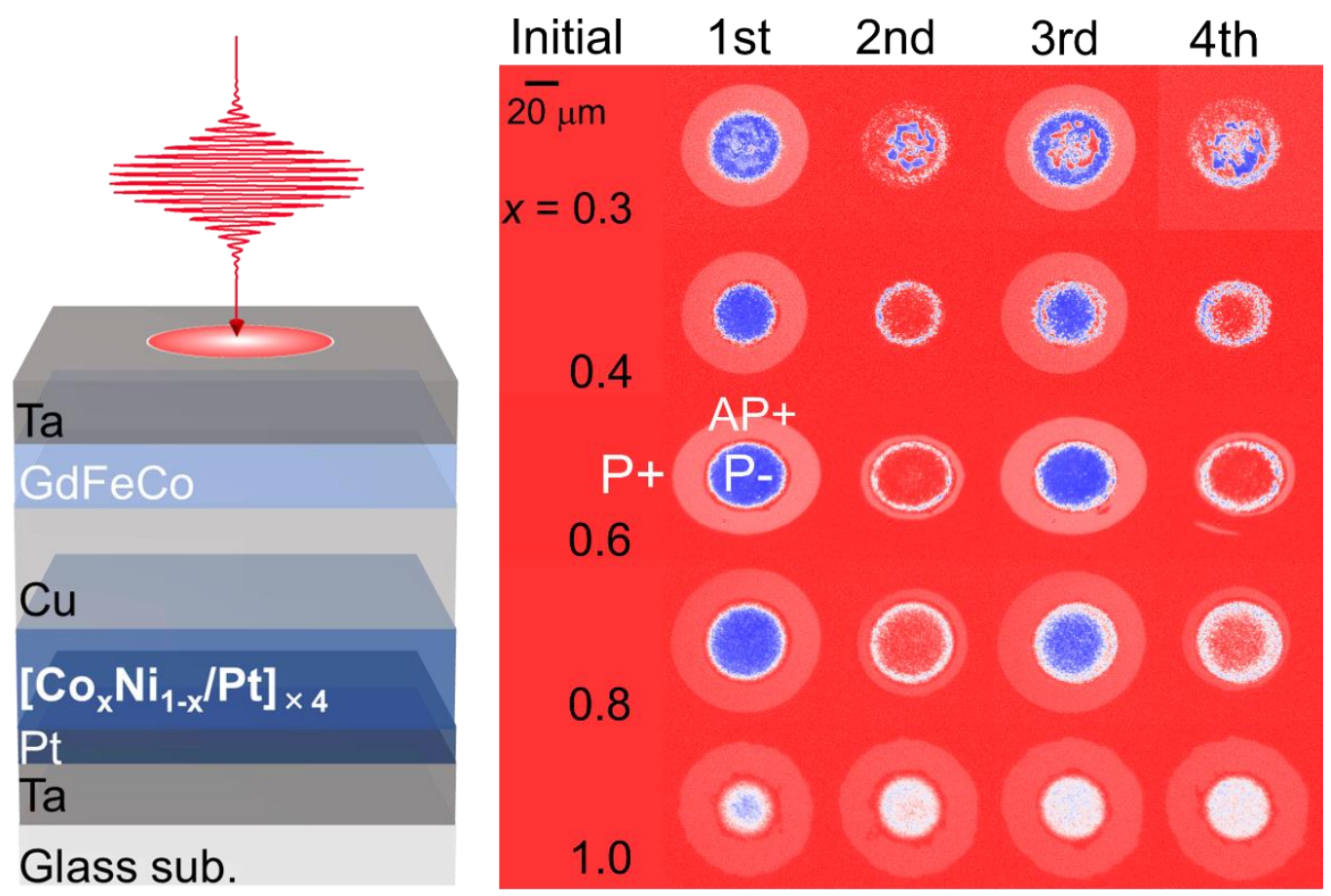


\section{MAIN TEXT}

Magnetization manipulation using ultrashort optical pulses without the use of any magnetic field, namely all-optical switching (AOS), has attracted much attention because of the potential application for ultrafast and energy-efficient magnetic storage or memory devices ${ }^{1-15}$. Two types of AOS have been observed. In the case of all-optical helicity-dependent switching (AO-HDS) magnetization can be switched by circularly polarized light pulses and the final magnetic state is determined by the light helicity. Recent experimental studies revealed that AO-HDS is observed for a large variety of magnetic materials such as rare earth (RE)-transition metal (TM) alloy and multilayers, RE-free synthetic ferrimagnetic heterostructures, ferromagnetic (FM) multilayers like $[\mathrm{Co} / \mathrm{Pt}]_{\mathrm{N}}$ and granular recording media ${ }^{2-5,16}$. However, unfortunately, multiple pulses are required to observe AO-HDS ${ }^{6}$, even if a drastic reduction of the number of pulses required has been recently shown ${ }^{17}$. In the case of all-optical helicity-independent switching (AO-HIS), the magnetization can be switched with one single femto-second pulse whatever light helicity is used. It has also been demonstrated using a single femto-second electron pulse ${ }^{18,19}$.

Nonetheless, AO-HIS is observed only for specific ferrimagnetic materials. First, it was demonstrated only for GdCo alloys and then for a series of Gd-based alloys and multilayers. The number of material exhibiting AO-HIS is limited even if it has recently been observed for TbCo ${ }^{20}$ and half-metallic compensated ferrimagnetic Heusler alloy $\mathrm{Mn}_{2} \mathrm{Ru}_{\mathrm{x}} \mathrm{Ga}$ with crystallographically-inequivalent Mn sublattices ${ }^{21}$. The mechanism behind AO-HIS still remains as a subject of intense debate ${ }^{8-13,20}$. In GdFeCo, it was attributed to the presence of two exchange coupled magnetic sublattices (the RE and the TM one) showing different relaxation times after ultrafast laser excitation. In this model, the toggle effect is mediated by the antiferromagnetic exchange coupling between the $\mathrm{Gd}$ and the $\mathrm{FeCo}^{8-13}$. 
In addition, AO-HIS had never been observed for a single ferromagnetic layer. However, single laser pulse switching has been demonstrated for a ferromagnetic $[\mathrm{Co} / \mathrm{Pt}]$ multilayers in an exchange coupled $\mathrm{GdFeCo} /[\mathrm{Co} / \mathrm{Pt}]$ bilayer as well as in $\mathrm{GdFeCo} / \mathrm{Cu} /[\mathrm{Co} / \mathrm{Pt}]$ spin-valve structure without exchange coupling between the two magnetic layers ${ }^{14,15}$. In the latter case, the $[\mathrm{Co} / \mathrm{Pt}]$ switching would result from the combined effect of the spin current generated by the AO-HIS of the GdFeCo layer and the laser-induced heating of the ferromagnet ${ }^{15}$.

From the later work it was suggested that a single femto-second spin current pulse can induce the switching of the magnetization of a ferromagnetic layer ${ }^{15}$, many questions remain unanswered. For instance, how much polarized current is needed? How much heat needs to be brought to the ferromagnetic layer? What type of ferromagnet can be switched? What are the key ferromagnet characteristics needed to observe the switching? Does the Curie temperature of the FM play a role in the switching? What sets the fluence and the pulse duration needed to observe the single pulse reversal?

In this study, we investigate the switching of $\left[\mathrm{Co}_{x} \mathrm{Ni}_{1-x} / \mathrm{Pt}\right]$ in $\mathrm{GdFeCo} / \mathrm{Cu} /\left[\mathrm{Co}_{x} \mathrm{Ni}_{1-x} / \mathrm{Pt}\right]$ spinvalve structure. $\left[\mathrm{Co}_{x} \mathrm{Ni}_{1-\mathrm{x}} / \mathrm{Pt}\right]$ is a model system showing perpendicular anisotropy with a Curie temperature $\left(T_{\mathrm{C}}\right)$ that can be tuned from $400 \mathrm{~K}$ to close $850 \mathrm{~K}$ by adjusting the $\mathrm{Co}_{\mathrm{x}} \mathrm{Ni}_{1-\mathrm{x}}$ concentration. From the experimental results and using a three temperature model, we can demonstrate that the ferromagnetic layer needs to be sufficiently demagnetized to ensure its switching when the spin current generated by the GdFeCo demagnetization reaches the FM layer. This highlights the fact that the FM Curie temperature has a significant role in the observed switching. 


\section{RESULTS}

\section{Film fabrication and Curie temperature}

$\mathrm{Ta}(5) / \mathrm{Gd}_{23.3} \mathrm{FeCo}_{76.7}(5) / \mathrm{Cu}(10) /\left[\mathrm{Co}_{\mathrm{x}} \mathrm{Ni}_{1-\mathrm{x}}(1) / \mathrm{Pt}(1)\right]_{4} / \mathrm{Pt}(3) / \mathrm{Ta}(5)$ stacks were deposited on glass substrate by dc/rf magnetron sputtering as shown in Figure 1a. The numbers in parentheses are nominal thicknesses in $\mathrm{nm}$. FeCo rich composition is used for the GdFeCo layer, meaning that net magnetization of the alloy is parallel to the magnetization of the FeCo sublattice. We prepared several samples with different Co compositions $\mathrm{x}(=0.2,0.3,0.4,0.6,0.8,1.0)$ in $\left[\mathrm{Co}_{\mathrm{x}} \mathrm{Ni}_{1-\mathrm{x}}\right]$ to tune its Curie temperature $T_{\mathrm{C}}{ }^{22}$. The temperature dependence of the magnetization of $\mathrm{Ta}(5) / \mathrm{Cu}(10) /\left[\mathrm{Co}_{\mathrm{x}} \mathrm{Ni}_{1-\mathrm{x}}(1) / \mathrm{Pt}(1)\right]_{4} / \mathrm{Pt}(3) / \mathrm{Ta}(5)$ was measured by a vibrating sample magnetometer with an oven. The increase of the $T_{\mathrm{C}}$ as the Co concentration increases in $\mathrm{Co}_{\mathrm{x}} \mathrm{Ni}_{1-\mathrm{x}}$ is shown in Figure 1b. From previous reports ${ }^{23,24}$, GdFeCo exhibits a Curie Temperature around $540 \mathrm{~K}$, which corresponds to the Curie temperature of a $\left[\mathrm{Co}_{\mathrm{x}} \mathrm{Ni}_{1-\mathrm{x}} / \mathrm{Pt}\right]$ multilayer for $\mathrm{x}$ between 0.3 and 0.4 . Figure $1 \mathrm{c}$ shows one example of the magneto-optic Kerr effect (MOKE) signal $\left(\theta_{\mathrm{K}}\right)$ as a function of the magnetic field $(H)$ applied perpendicularly to the film plane for the sample with $\mathrm{x}=0.6$. We confirm that four magnetic configurations $(\mathrm{P}+, \mathrm{AP}+, \mathrm{AP}-, \mathrm{P}-)$ can be accessed by applying a field except for the sample with $\mathrm{x}=0.2$. The four magnetic configurations are the one for which the two magnetization configurations are parallel $\mathrm{P}+, \mathrm{P}-$, or anti-parallel $\mathrm{AP}+$, AP-. $\mathrm{P}+$ and $\mathrm{AP}+$ correspond to the cases for which the magnetization of the $\left[\mathrm{Co}_{\mathrm{x}} \mathrm{Ni} i_{1-\mathrm{x}} / \mathrm{Pt}\right]$ is along the positive field direction. Red and blue triangle symbols in Fig. 1c are minor loops corresponding to the magnetization reversal of the GdFeCo layer. No shift of the minor loop indicates no coupling between $\mathrm{GdFeCo}$ and $\left[\mathrm{Co}_{x} \mathrm{Ni}_{1-\mathrm{x}} / \mathrm{Pt}\right]$ magnetic layers (The results for other $\mathrm{x}$ are shown in supplementary Supporting Information S2). 

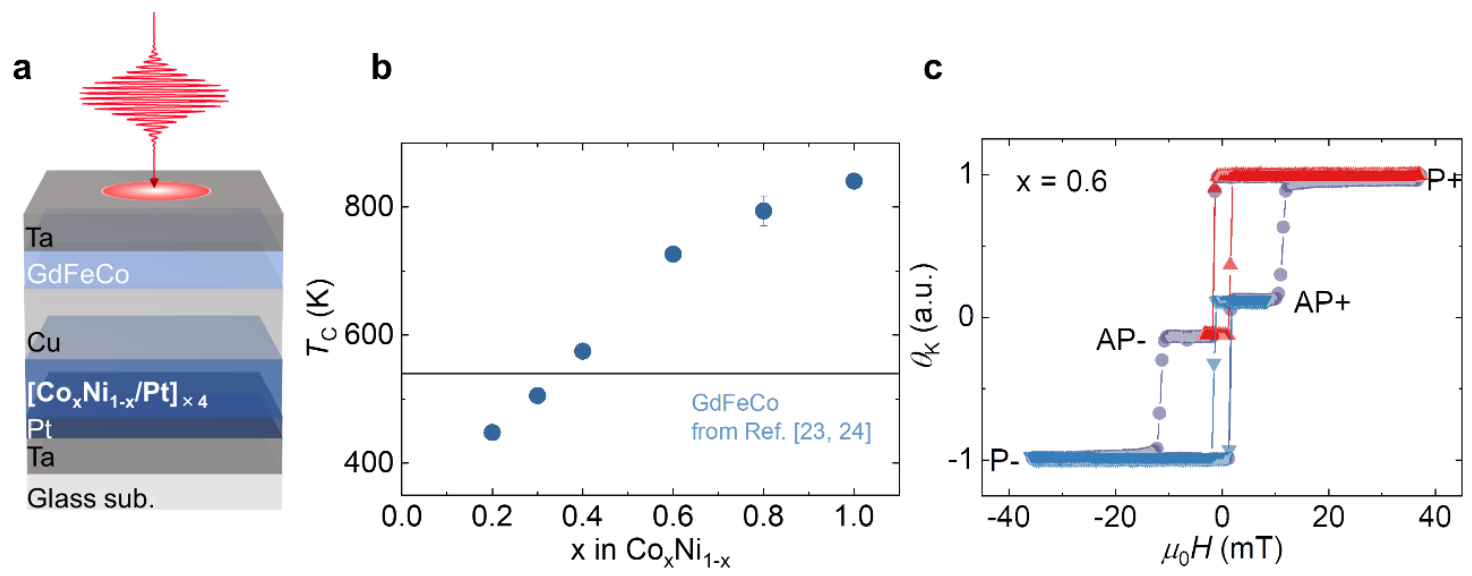

Figure 1. Stacking structure and magnetic properties. (a). Stacking structure for the spin-valve structure for AOS measurement. (b). Curie temperature measured for $\left[\mathrm{Co}_{\mathrm{x}} \mathrm{Ni}_{1-\mathrm{x}} / \mathrm{Pt}\right]$ multilayers plotted as a function x (blue symbol). The line corresponds to $T_{\mathrm{C}}$ for GdFeCo (Ref. [23, 24]). (c). MOKE hysteresis loop measured from film side for $\left[\mathrm{Co}_{0.6} \mathrm{Ni}_{0.4} / \mathrm{Pt}\right] / \mathrm{Cu} / \mathrm{GdFeCo}$ spin-valve structure.

\section{All-optical switching}

Figure 2a shows MOKE images obtained after shooting one, then two, three, and four single femto-second laser pulses starting from a saturated $\mathrm{P}+$ state for different samples. Here, the sample is irradiated from the capping layer side and MOKE images are obtained from the substrate side. The fluence used in the measurements shown in the figure are between 14 and 21 $\mathrm{mJ} / \mathrm{cm}^{2}$. The laser pulse duration is fixed to $35 \mathrm{fs}$. When the MOKE images are changed from dark red $(\mathrm{P}+)$ to light red $(\mathrm{AP}+)$ it indicates that only the $\mathrm{GdFeCo}$ layer magnetization has been switched. This transition is observed, for an outer ring, after a single pulse for all samples concentration. After every pulse the GdFeCo magnetization switches. This is an AO-HIS. The change of MOKE contrast from dark red $(\mathrm{P}+)$ to dark blue $(\mathrm{P}-)$ proves that both the GdFeCo and the $\left[\mathrm{Co}_{x} \mathrm{Ni}_{1-\mathrm{x}} / \mathrm{Pt}\right]$ magnetization are switched. The later is observed after one single pulse in the case of $x=0.4,0.6,0.8$. For those samples, the single pulse reversal of both layers is possible as 
already observed by Iihama et al. ${ }^{15}$. This effect can be explained by the generation of a spin current pulse, whose polarization is parallel to the initial Gd magnetization, due to the change in magnetization (-d $M / \mathrm{d} t)$ generated by the GdFeCo layer AO-HIS. The generated spin current can then switch the $\left[\mathrm{Co}_{\mathrm{x}} \mathrm{Ni}_{1-\mathrm{x}} / \mathrm{Pt}\right]$. We also observe a white spot at the center at high power, corresponding to the formation of random domain states due to high energy density (Shown in Supporting Information S3, and ref. (15)). In the case of $\mathrm{x}=1.0$, although both layers are impacted by the laser beam, no full switching of the layers is observed. To determine the laser pulse energy $E_{\mathrm{p}}$ required for switching, the radius of the switched domain $r_{\mathrm{i}}$ (where $\mathrm{i}$ indicates the type of reversal) is evaluated (see Supporting Information S3). Assuming a Gaussian spatial distribution of the laser pulse as follow: $F_{\mathrm{pi}}=2 E_{\mathrm{p}} /\left(\pi w_{0}^{2}\right) \exp \left(-2 r_{\mathrm{i}}^{2} / w_{0}^{2}\right)$, where $F_{\mathrm{pi}}$ and $2 w_{0}$ are the threshold fluence and the $1 / e^{2}$ spot size respectively (details are shown in Supporting Information $\mathrm{S} 3)$. Figure $2 \mathrm{~b}$ shows the obtained $F_{\mathrm{pi}}$ versus $T_{\mathrm{C}}$. We do not observe any effect of the FM composition on the critical fluence required to switch the GdFeCo layer. On the other hand, the threshold fluence for the switching of both layers $\left(F_{\text {both }}\right)$ and the generation of multi-domain states $\left(F_{\text {multi }}\right)$ increases with the increasing $T_{\mathrm{C}}$ of the FM layer. Figure $2 \mathrm{c}$ shows the difference between the fluence to obtain the multi-domain state and the fluence to switch both layers ( $F_{\text {multi }}$ - $\left.F_{\text {both }}\right)$ as a function of $T_{\mathrm{C}} . F_{\text {multi }}-F_{\text {both }}$ increases with increasing $T_{\mathrm{C}}$ up to $\sim 800 \mathrm{~K}$, indicating that there is a certain fluence window that allows the switching of both layers without generating a multi-domain state. Note that other parameters such as saturation magnetization, perpendicular anisotropy, refractive indices, moreover, the probability of spin-flipping, also depends on $\mathrm{x}$ and may affect the FM layer switching since it changes the amount of angular momentum transfer to the magnetization (see Supporting Information S1, S4, and S5). This latter comment is out of the 
scope of this study. In Figure 2, calculated fluence to switch the interface layer ( $\left.F_{\text {int }}\right)$ and to fully demagnetize at the end of the FM layer $\left(F_{\text {end }}\right)$ are shown. These will be discussed later.

a

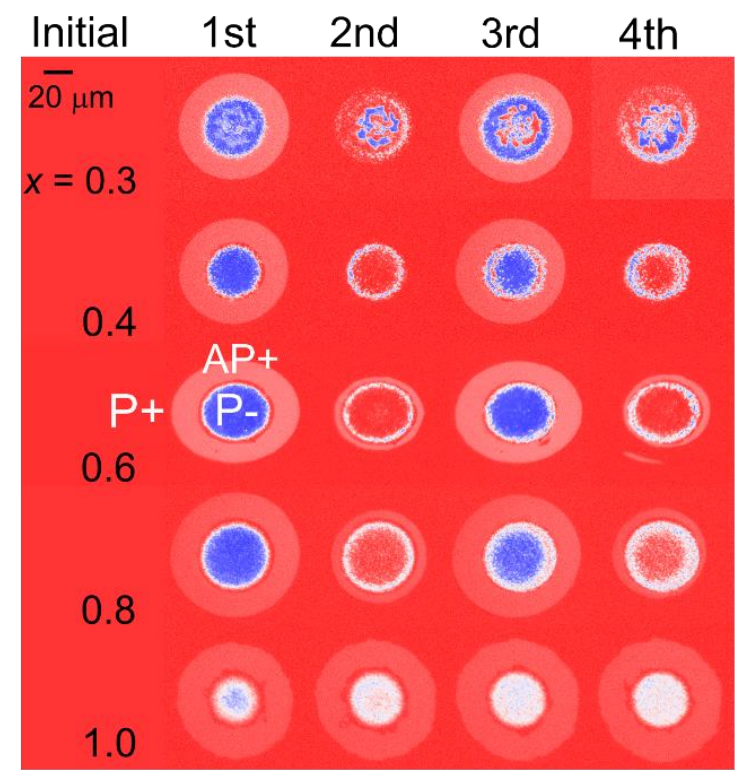

b

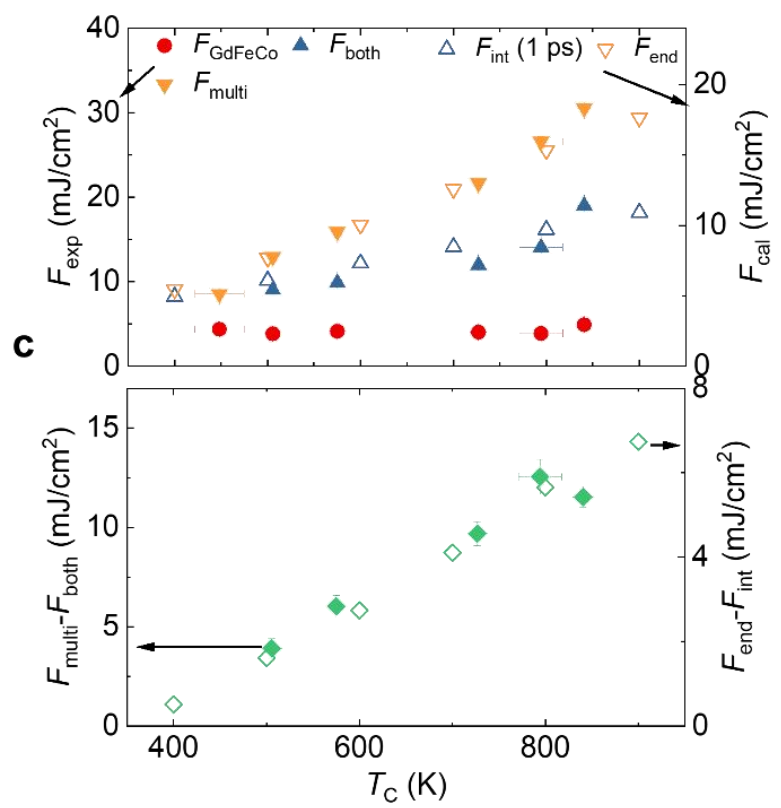

Figure 2. All-optical magnetization switching for ferromagnetic materials with different Curie temperatures. (a). MOKE images obtained after irradiation of 1 then 2, 3, and 4 fs-laser pulses starting from a $\mathrm{P}+$ initial sate. (b). Threshold fluences for reversing the GdFeCo magnetization only (circle symbols), reversing the magnetization of both the GdFeCo and the ferromagnetic layers (triangle symbols), and multi-domain states (inverted triangle symbols) are plotted as a function of the FM layer Curie temperature $\left(T_{\mathrm{C}}\right)$. Closed (resp. Opened) symbols correspond to the experiment (resp. calculation). The equilibrium temperature is defined as the temperature reached by the system after 4.7 ps when the 3 baths are at equilibrium i.e at the same temperature. Equilibrium temperature of $643 \mathrm{~K}$ is calculated by a microscopic three temperature model for a $10 \mathrm{~mJ} / \mathrm{cm} 2$ fluence for an $\mathrm{x}$ of $0.6\left(T_{\mathrm{C}}=700 \mathrm{~K}\right)$. (c). Difference in the threshold fluence for 
switching both layer $\left(F_{\text {both }}\right)$ and multi-domain states $\left(F_{\text {multi }}\right)$ are plotted as a function of $T_{\mathrm{C}}$. Closed (Opened) symbols correspond to the experiment (calculation).

Next, we study the influence of the thickness of the first Co layer at the interface with the $\mathrm{Cu}$ layer on the single pulse switching of the FM layer. Note that it is the part of the ferromagnetic layer interacting first with the electrons coming from the GdFeCo layer. We measured the sample magnetic state after a few single pulses for samples with different interface Co layer thicknesses $t_{\mathrm{Co}}$ in the following stack: $\mathrm{Ta}(5) / \mathrm{Gd}_{23.3} \mathrm{FeCo}_{76.7}(5) / \mathrm{Cu}(10) / \mathrm{Co}\left(t_{\mathrm{Co}}\right) / \mathrm{Pt}(1) /[\mathrm{Co}$ $(0.6) / \mathrm{Pt} \mathrm{(1)}]_{3} / \mathrm{Pt}(3) / \mathrm{Ta}(5) /$ glass sub. (Figure 3a). Here, the laser pulse duration is fixed to $\sim 35$ fs. Figure $3 \mathrm{~b}$ shows MOKE images after irradiation of fs-laser pulses for the samples with different $t_{\mathrm{Co}} . F_{\mathrm{p}}$ values are evaluated using the method described above and plotted as a function of $t_{\mathrm{Co}}$ as shown in Figure 3c. The threshold fluence of GdFeCo is still constant and around $\sim 5 \mathrm{~mJ} / \mathrm{cm}^{2}$. On the other hand, the threshold fluence for the switching of both $\mathrm{GdFeCo}$ and $[\mathrm{Co} / \mathrm{Pt}]$ layers increases with increasing $t_{\mathrm{Co}}$. The above results indicate that the interface layer plays a major role in the single pulse switching mechanism.

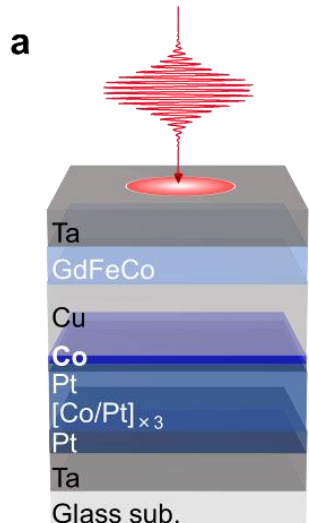

Glass sub.
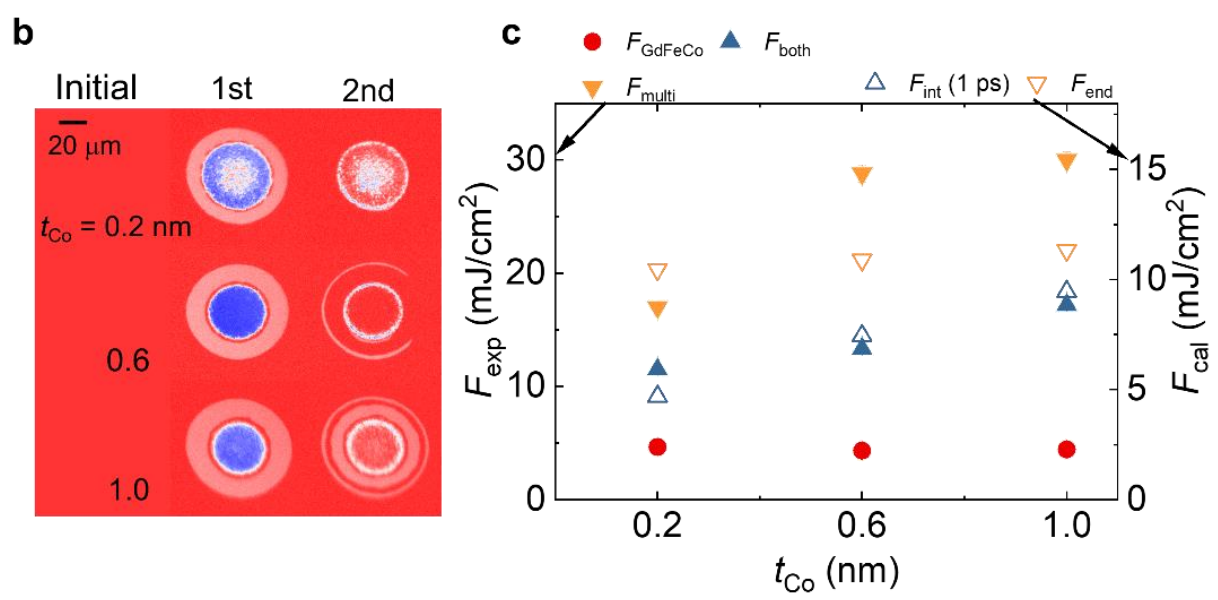
Figure 3. All-optical magnetization switching experiment by engineering thickness of interface Co layer. (a). Stacking structure used for this study. (b). MOKE images after irradiation of fs-laser pulses with different interface Co layer thickness. Irradiated fluence is $16 \sim 18 \mathrm{~mJ} / \mathrm{cm}^{2}$. (c). Threshold fluences for reversing the magnetization of only GdFeCo layer (circle symbols), reversing the magnetization of both GdFeCo and FM layers (triangle symbols), and multidomain states (inverted triangle symbols) are plotted as a function of interface Co layer thickness. Closed (Opened) symbols correspond to the experiment (calculation).

Finally, we performed single pulse switching measurements for various pulse durations ranging from 35 fs to $3 \mathrm{ps}$. The sample is the same as in the previous experiment with $t_{\mathrm{Co}}=0.6$ nm. Figure 4a shows MOKE images after irradiation of laser pulse with different pulse durations. Clear single-shot switching of the $[\mathrm{Co} / \mathrm{Pt}]$ layer is observed for a pulse duration of up to $500 \mathrm{fs}$. Note that in the case of a single-layer GdFeCo, single-shot AO-HIS up to $1 \sim 10$ ps was observed depending on the Gd composition ${ }^{24}$. Figure $4 \mathrm{~b}$ shows the threshold fluence for the $\mathrm{GdFeCo}$ switching $\left(F_{\mathrm{GdFeCo}}\right)$, both layers switching $\left(F_{\text {both }}\right)$ and formation of multi-domain states $\left(F_{\text {multi }}\right)$ plotted as a function of the laser pulse duration. For the GdFeCo switching and both layers switching, the threshold fluence does not change when increasing the pulse duration up to $200 \mathrm{fs}$, then it increases with increasing the pulse duration up to 1(3) ps for $F_{\mathrm{both}}\left(F_{\mathrm{GdFeC}}\right)$. On the other hand, the evolution of $F_{\text {multi }}$ as a function of the pulse duration is different from the evolution of $F_{\text {both }}$ and $F_{\mathrm{GdFeC} .} . F_{\text {multi }}$ is constant for pulse durations up to $100 \mathrm{fs}$, then it starts to decrease with increasing pulse duration up to $2 \mathrm{ps}$. 


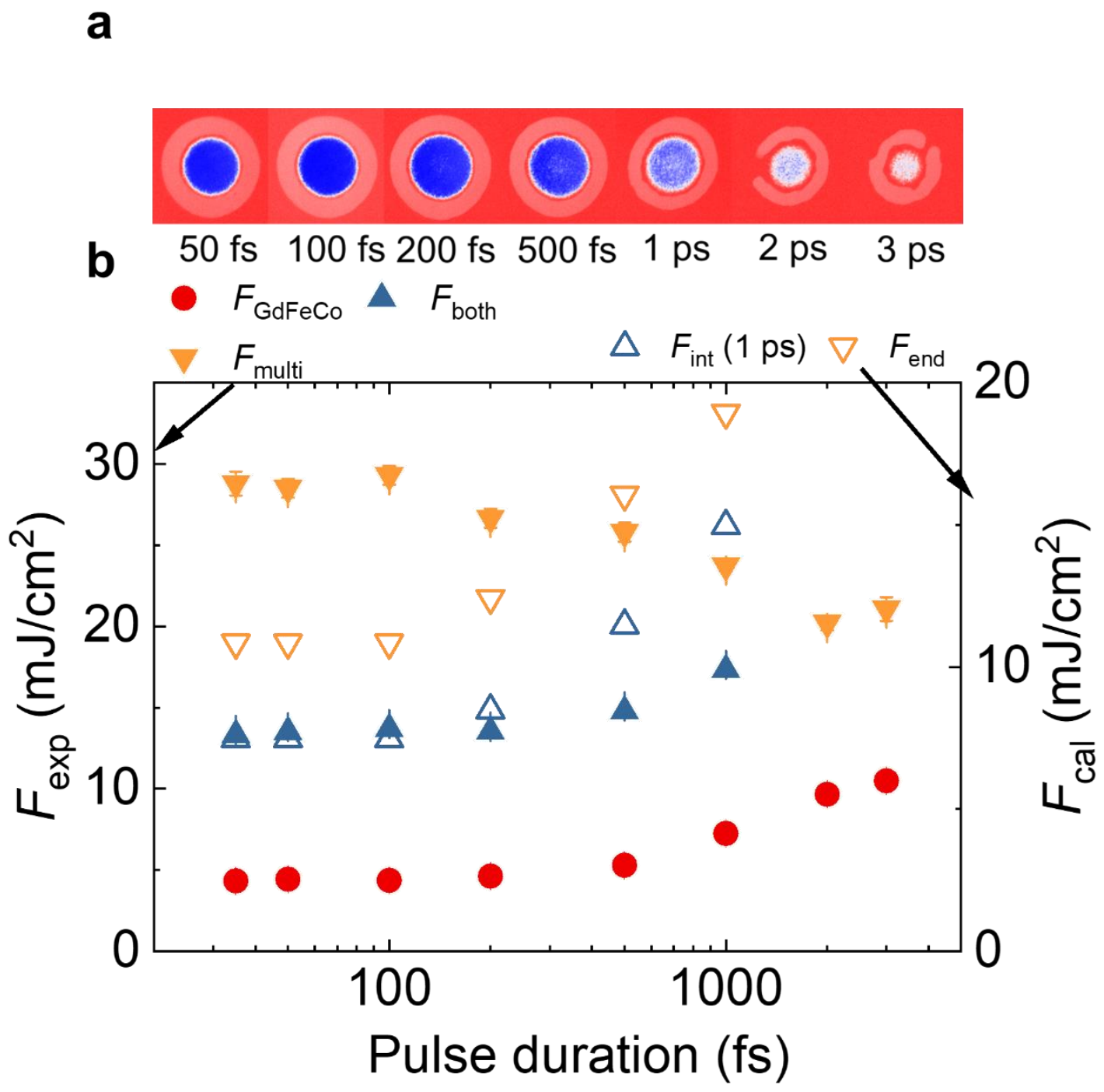

Figure 4. All-optical magnetization switching experiment on spin-valve structure with different optical pulse duration. (a). MOKE images obtained after irradiation of one single laser pulses with a different optical pulse duration ranging from 50 fs to 3 ps. (b). Threshold fluences for reversing the magnetization of the GdFeCo layer only (circle symbols), for reversing the magnetization of both the GdFeCo and the ferromagnetic layer (triangle symbols), and forming multi-domain states (inverted triangle symbols) are plotted as a function of pulse duration. Closed (resp. Opened) symbols correspond to the experiment (resp. calculation). 


\section{DISCUSSION}

We have demonstrated in this work that three parameters can have a strong impact on the single pulse switching of a ferromagnetic multilayer $\left[\mathrm{Co}_{\mathrm{x}} \mathrm{Ni}_{1-\mathrm{x}} / \mathrm{Pt}\right]$ in a $\mathrm{GdFeCo} / \mathrm{Cu} /\left[\mathrm{Co}_{\mathrm{x}} \mathrm{Ni}_{1-\mathrm{x}} / \mathrm{Pt}\right]$ spin-valve: the concentration of the CoNi alloys, the thickness of the interface ferromagnetic layer in contact with the $\mathrm{Cu}$ and the laser pulse duration.

From those measurements, one can reach the following general understanding of the mechanism behind the two-layer switching with one single light pulse: The laser pulse interacts with the GdFeCo leading to its switching, this is AO-HIS. During the reversal, the magnetization relaxation of the two sublattices leads to two distinct spin current pulses separated in time as described by Choi et al $^{25}$. The first one is generated mainly by the TM sublattice, a few femtoseconds after the laser pulse, and is polarized along the TM magnetization direction. The second one is generated and polarized by the Gd after $1 \mathrm{ps.} \mathrm{We} \mathrm{believe} \mathrm{that} \mathrm{in} \mathrm{order} \mathrm{for} \mathrm{the} \mathrm{FM} \mathrm{layer} \mathrm{to}$ switch, this second spin current pulse has to interact with the FM layer when the FM is sufficiently demagnetized. Based on angular momentum conservation, our hypothesis is that the FM switching will take place only if the remaining magnetization of the interfacial FM layer reaches a threshold value which corresponds to the amount of angular momentum transferred by the spin current originating from the Gd sublattice. We do not consider the first current pulse as it interacts with the FM layer while its demagnetization has barely started. However, the second pulse reaches the FM layer typically 1 ps after the FM started demagnetizing. The demagnetization is mainly generated by the direct laser irradiation plus possibly by some hot electrons generated in the structure ${ }^{26}$. To observe the multi-domain state, we presume that the 
full $\mathrm{CoNi} / \mathrm{Pt}$ layer needs to be demagnetized for a long period such that the system can no longer "remember" the polarization of the Gd polarized current.

The influence of the CoNi concentration on the fluence needed for the ferromagnetic switching and the multi-domain formation is then consistently explained by the fact that demagnetized states can be reached with less energy as the $T_{\mathrm{C}}$ of the FM layer is lower. Similarly, the significant change in the fluence needed to switch a $\mathrm{Co} / \mathrm{Pt}$ multilayer as a function of the thickness of the interface Co layer deposited next to the $\mathrm{Cu}$ layer can be explained with a similar argument. In a $\mathrm{Co} / \mathrm{Pt}$ multilayer, the exchange coupling inside the Co layer is strong and it is weaker between the Co layers because of the Pt layer ${ }^{27}$. The exchange coupling inside the Co layer also depends on its thickness ${ }^{16}$. If the interface Co layer is thin, the effective exchange coupling inside the layer will be weak and it will require less energy to be partly demagnetized. It will then be easier to reach the demagnetization threshold and consequently to be switched. If the interface layer switches, the switching of the rest of the multilayer can then be explained by the propagation of the reversal due to the exchange coupling between the layer as observed in several systems ${ }^{14,28}$.

Concerning the experiments for which the laser pulse duration is tuned, we observed that the fluence needed for the GdFeCo switching increases monotonically as the pulse duration increases, as has been reported previously ${ }^{24,29}$. In our spin-valve samples, the threshold for switching both the GdFeCo and the ferromagnet's magnetization follows a similar trend of monotonic increase. Both these increases can be simply explained by the fact that, as the pulse duration becomes longer more energy is lost to the lattice that does not contribute to the demagnetization or the switching. Surprisingly, the threshold energy to generate a multi-domain state decreases with increasing pulse duration, which cannot be understood in the frame of the 
above discussion. This has never been reported in single GdFeCo layers ${ }^{24}$ and is certainly due to our spin-valve structure. We believe thus that such decrease in threshold fluence with pulse duration maybe due to (mutual) spin currents (stronger for short pulses) that stabilize the magnetization of the remote layer.

In order to be more quantitative, we first evaluate the amount of angular momentum or magnetic moment transfer from the $\mathrm{GdFeCo}$ (during its reversal) to the $\mathrm{CoNi} / \mathrm{Pt}$ multilayer. Considering Choi et al ${ }^{25}$ work, we can assume that the change in magnetization at the $\mathrm{Cu} / \mathrm{FM}$ interface, due to the generated spin accumulation, can reach a maximum value of about $\Delta M=$ $1000 \mathrm{~A} / \mathrm{m}$ at $1 \mathrm{ps}$ since the laser pulse we are using is ten time more energetic. This $\Delta M$ value is a very rough estimate since we consider that the same magnetization is transferred to the first layer of the ferromagnetic multilayer. Moreover, we do not take into account the time dependence of the angular momentum transfer and the nonuniformity of the resulting magnetization in the first CoNi layer. We can now define a criterion to observe the switching of the FM multilayer: "To generate the switching of the FM, the magnetization of the first CoNi layer at the interface with $\mathrm{Cu}$ should reach the value $\Delta M 1 \mathrm{ps}$ after the laser pulse irradiation".

We then assume that after the first layer has switched the rest of the layer will reverse because of the exchange coupling as in ref. (13), (14). Note that with the above criterion, switching is observed when the normalized magnetization of the interface layer $m=M / M_{\mathrm{S}}$ reaches around $10^{-}$ 3. A better model would consider the rate of transfer of angular momentum through an s-d model ${ }^{30}$, although this is out of the scope of this study. The criterion for the appearance of the multidomain state should be the complete demagnetization of the full multilayer. However, because a 
zero magnetization depends on the numerical precision and because it does not lead to qualitative changes, we assume that it is the case when the magnetization of the last layer of the FM reaches $10 \%$ of $\Delta M$.

Then, in order to determine the time evolution of $\mathrm{m}$ at the interfacial CoNi layer and in the last CoNi layer, due to the laser pulse, we have modeled the magnetization dynamics of the FM layer with a microscopic three temperature model (M3TM) for a spin $1 / 2$ ferromagnetic system, considering heat diffusion and realistic light absorption profiles in the full structure. Information on the calculation procedure can be found in Supporting Information S5 ${ }^{31}$. Several parameters used for the calculation, shown in the Supporting Information, are determined either experimentally or from the literature ${ }^{24,26,32}$. Using the above criterion, we can then calculate the fluence to switch the interface layer $F_{\text {int }}$ and compare with the experimentally determined fluence needed to switch both layers $\left(F_{\text {both }}\right)$. Similarly, the calculated fluence to demagnetize the last FM $F_{\text {end }}$ is compared to the experimentally determined fluence needed to observe a multi-domain state $\left(F_{\text {multi }}\right)$ as shown in Figure $2 \mathrm{~b}, 3 \mathrm{c}$ and $4 \mathrm{~b}$.

In Figure $2 \mathrm{~b}$, one can see the evolution of the calculated fluences $\left(F_{\text {end }}\right.$ and $\left.F_{\text {int }}\right)$ as a function of the CoNi Curie temperature. We can see that the calculation reproduces accurately the experimental trend. With no adjustment, we find about a factor two difference, which is reasonably good for such a simple model. In Figure $3 \mathrm{c}$ The evolution of the fluence as a function of the interface Co layer thickness is also well reproduced and the same factor two is observed. In Figure $4 \mathrm{~b}$, showing the evolution of the fluence for both layer switching $\left(F_{\text {both }}\right.$ and $\left.F_{\text {int }}\right)$ as a function of the pulse duration, a good agreement is observed between calculations and experiments. However, the evolution of the multi-domain fluence $\left(F_{\text {end }}\right.$ and $\left.F_{\text {multi }}\right)$ as a function of the pulse duration is following the opposite trend. This could be a hint that the spin current 
from the GdFeCo demagnetization not only induce the FM switching but tends to avoid its demagnetization. Indeed if the laser pulse is longer, we expect the spin current to decrease because the GdFeCo relaxation will be longer (-dM/dt will decrease). Consequently, the fluence needed for the switching of both layers should increase and this spin current should no longer be as efficient in maintaining the magnetization direction once it has switched. Thus, the fluence needed to observe the multi-domain state should decrease.

In summary, we have studied femto-second single-shot all-optical magnetization switching for the various ferromagnetic layers $(\mathrm{FM})$ in $\mathrm{GdFeCo} / \mathrm{Cu} / \mathrm{FM}$ spin valves and various pulse durations. The results shed light on the mechanism leading to this single laser pulse switching of a ferromagnet where FM layer thickness, Curie temperature, and pulse duration play decisive roles. Based on this finding, we developed a phenomenological criterion, and confirmed that it reproduces most of the experimental results. We also find that AOS of ferromagnetic layer is observed in a wide range of stack structures and pulse duration ranging from 35 fs to $1 \mathrm{ps,}$ indicating robustness of the phenomenon. This work will provide us a way to engineer ferromagnetic materials for single-shot all-optical magnetization switching.

\section{MATERIALS AND METHODS}

Sample Preparation

All samples were prepared by physical vapor deposition. The base pressure used to deposit multilayer film was about $1 \times 10^{-7}$ Torr. Basic stacking structures used in this study for the spinvalve structure were as follows: 
$\mathrm{Ta}(5) / \mathrm{Gd}_{23.3} \mathrm{FeCo}_{76.7}(5) / \mathrm{Cu}(10) /\left[\mathrm{Co}_{\mathbf{x}} \mathrm{Ni}_{1-\mathrm{x}}(1) / \mathrm{Pt}(1)\right]_{4} /$

$\mathrm{Pt}$ (3)/Ta (5)/glass sub. (thickness in $\mathrm{nm}$ ).

The different composition $\mathrm{x}$ of Co were $0.2,0.3,0.4,0.6,0.8$ and 1.0.

$\mathrm{Ta}(5) / \mathrm{Gd}_{23.3} \mathrm{FeCo}_{76.7}(5) / \mathrm{Cu}(10) / \mathrm{Co}\left(t_{\mathrm{Co}}\right) / \mathrm{Pt}(1) /[\mathrm{Co}(0.6) / \mathrm{Pt}(1)]_{3} / \mathrm{Pt}(3) / \mathrm{Ta}(5) / \mathrm{glass}$ sub. The different top Co thicknesses $t_{\mathrm{Co}}$ in $[\mathrm{Co} / \mathrm{Pt}]$ were $0.2,0.6$ and $1.0 \mathrm{~nm}$.

\section{AOS Measurement}

Ti: sapphire fs-laser source and regenerative amplifier were used for the pump laser beam in AOS measurement. Wavelength and repetition rate of the fs laser was $800 \mathrm{~nm}$ and $5 \mathrm{kHz}$, respectively. The $1 / e^{2}$ spot size $2 w_{0}$ is $\approx 94 \mu \mathrm{m}$. MOKE images were obtained from the other side of the film. LED light source with a wavelength of $628 \mathrm{~nm}$ was used for taking MOKE images.

\section{ASSOCIATED CONTENT}

\section{Supporting Information.}

The Supporting Information is available free of charge at XXX.

Magnetic properties of $\left[\mathrm{Co}_{\mathrm{x}} \mathrm{Ni}_{1-\mathrm{x}} / \mathrm{Pt}\right]$, MOKE Hysteresis loop, Determination of threshold

fluence, Absorption profile, and M3TM calculation for the spin-valve structure. (PDF)

\section{AUTHOR INFORMATION}




\section{Corresponding Author}

* Stéphane Mangin - stephane.mangin@univ-lorraine.fr

\section{Author Contributions}

J.I., S.I., and S.M. planned study. M.H. performed sample fabrication. J.I., S.I., and Q.R. performed experiments, G.M. and J.G. helped for measurement. J.I. analyzed data. J.I., Q.R., and J.H. performed the calculation. J.I., Q.R., S.I., and S.M. wrote the manuscript with inputs from G.M., J.G., and S.F. All authors contributed to discuss the measurement results.

\section{Notes}

The authors declare no competing financial interest.

\section{ACKNOWLEDGMENT}

The authors thank T. Ferté for technical support. This work was supported in part by JSTOPERA, JSPS KAKENHI (JP19J12926), DIARE, the French PIA project "Lorraine Université d'Excellence" reference ANR-15-IDEX-04-LUE, the Institut Carnot ICEEL, the Sakura Program, the JSPS Bilateral Program and the Tohoku University-Université de Lorraine Matching Funds. This work is part of the project PLUS supported by the "FEDER-FSE Lorraine et Massif Vosges 2014-2020”, a European Union Program. J.I. acknowledges support from GP-Spin.

\section{REFERENCES}

(1) Kimel, A. V.; Li, M. Writing Magnetic Memory with Ultrashort Light Pulses. Nat. Rev. Mater. 2019, 4 (3), 189-200. https://doi.org/10.1038/s41578-019-0086-3.

(2) Stanciu, C. D.; Hansteen, F.; Kimel, A. V.; Kirilyuk, A.; Tsukamoto, A.; Itoh, A.; Rasing, Th. All-Optical Magnetic Recording with Circularly Polarized Light. Phys. Rev. Lett. 2007, 99 (4), 047601. https://doi.org/10.1103/PhysRevLett.99.047601. 
(3) Alebrand, S.; Gottwald, M.; Hehn, M.; Steil, D.; Cinchetti, M.; Lacour, D.; Fullerton, E. E.; Aeschlimann, M.; Mangin, S. Light-Induced Magnetization Reversal of High-Anisotropy TbCo Alloy Films. Appl. Phys. Lett. 2012, 101 (16), 162408. https://doi.org/10.1063/1.4759109.

(4) Mangin, S.; Gottwald, M.; Lambert, C.-H.; Steil, D.; Uhlír, V.; Pang, L.; Hehn, M.; Alebrand, S.; Cinchetti, M.; Malinowski, G.; Fainman, Y.; Aeschlimann, M.; Fullerton, E. E. Engineered Materials for All-Optical Helicity-Dependent Magnetic Switching. Nature Mater. 2014, 13 (3), 286-292. https://doi.org/10.1038/nmat3864.

(5) Lambert, C.-H.; Mangin, S.; Varaprasad, B. S. D. C. S.; Takahashi, Y. K.; Hehn, M.; Cinchetti, M.; Malinowski, G.; Hono, K.; Fainman, Y.; Aeschlimann, M.; Fullerton, E. E. AllOptical Control of Ferromagnetic Thin Films and Nanostructures. Science 2014, 345 (6202), 1337-1340. https://doi.org/10.1126/science.1253493.

(6) El Hadri, M. S.; Pirro, P.; Lambert, C.-H.; Petit-Watelot, S.; Quessab, Y.; Hehn, M.; Montaigne, F.; Malinowski, G.; Mangin, S. Two Types of All-Optical Magnetization Switching Mechanisms Using Femtosecond Laser Pulses. Phys. Rev. B 2016, 94 (6), 064412. https://doi.org/10.1103/PhysRevB.94.064412.

(7) Gorchon, J.; Yang, Y.; Bokor, J. Model for Multishot All-Thermal All-Optical Switching in Ferromagnets. Phys. Rev. B 2016, $94 \quad 020409$. https://doi.org/10.1103/PhysRevB.94.020409.

(8) Radu, I.; Vahaplar, K.; Stamm, C.; Kachel, T.; Pontius, N.; Dürr, H. A.; Ostler, T. A.; Barker, J.; Evans, R. F. L.; Chantrell, R. W.; Tsukamoto, A.; Itoh, A.; Kirilyuk, A.; Rasing, T.; Kimel, A. V. Transient Ferromagnetic-like State Mediating Ultrafast Reversal of Antiferromagnetically Coupled Spins. Nature 2011, $472 \quad$ (7342), 205-208. https://doi.org/10.1038/nature09901.

(9) Ostler, T. A.; Barker, J.; Evans, R. F. L.; Chantrell, R. W.; Atxitia, U.; ChubykaloFesenko, O.; El Moussaoui, S.; Le Guyader, L.; Mengotti, E.; Heyderman, L. J.; Nolting, F.; Tsukamoto, A.; Itoh, A.; Afanasiev, D.; Ivanov, B. A.; Kalashnikova, A. M.; Vahaplar, K.; Mentink, J.; Kirilyuk, A.; Rasing, T.; Kimel, A. V. Ultrafast Heating as a Sufficient Stimulus for Magnetization Reversal in a Ferrimagnet. Nat. Commun. 2012, 3 (1), 1-6. https://doi.org/10.1038/ncomms1666.

(10) Bergeard, N.; López-Flores, V.; Halté, V.; Hehn, M.; Stamm, C.; Pontius, N.; Beaurepaire, E.; Boeglin, C. Ultrafast Angular Momentum Transfer in Multisublattice Ferrimagnets. Nat. Commun. 2014, 5 (1), 3466. https://doi.org/10.1038/ncomms4466.

(11) Graves, C. E.; Reid, A. H.; Wang, T.; Wu, B.; de Jong, S.; Vahaplar, K.; Radu, I.; Bernstein, D. P.; Messerschmidt, M.; Müller, L.; Coffee, R.; Bionta, M.; Epp, S. W.; Hartmann, R.; Kimmel, N.; Hauser, G.; Hartmann, A.; Holl, P.; Gorke, H.; Mentink, J. H.; Tsukamoto, A.; Fognini, A.; Turner, J. J.; Schlotter, W. F.; Rolles, D.; Soltau, H.; Strüder, L.; Acremann, Y.; Kimel, A. V.; Kirilyuk, A.; Rasing, Th.; Stöhr, J.; Scherz, A. O.; Dürr, H. A. Nanoscale Spin Reversal by Non-Local Angular Momentum Transfer Following Ultrafast Laser Excitation in Ferrimagnetic GdFeCo. Nature Mater. 2013, 12 (4), 293-298. https://doi.org/10.1038/nmat3597. 
(12) Wienholdt, S.; Hinzke, D.; Carva, K.; Oppeneer, P. M.; Nowak, U. Orbital-Resolved Spin Model for Thermal Magnetization Switching in Rare-Earth-Based Ferrimagnets. Phys. Rev. B 2013, 88 (2), 020406. https://doi.org/10.1103/PhysRevB.88.020406.

(13) Lalieu, M. L. M.; Peeters, M. J. G.; Haenen, S. R. R.; Lavrijsen, R.; Koopmans, B. Deterministic All-Optical Switching of Synthetic Ferrimagnets Using Single Femtosecond Laser Pulses. Phys. Rev. B 2017, 96 (22), 220411. https://doi.org/10.1103/PhysRevB.96.220411.

(14) Gorchon, J.; Lambert, C.-H.; Yang, Y.; Pattabi, A.; Wilson, R. B.; Salahuddin, S.; Bokor, J. Single Shot Ultrafast All Optical Magnetization Switching of Ferromagnetic Co/Pt Multilayers. Appl. Phys. Lett. 2017, 111 (4), 042401. https://doi.org/10.1063/1.4994802.

(15) Ihama, S.; Xu, Y.; Deb, M.; Malinowski, G.; Hehn, M.; Gorchon, J.; Fullerton, E. E.; Mangin, S. Single-Shot Multi-Level All-Optical Magnetization Switching Mediated by Spin Transport. Adv. Mater. 2018, 30 (51), 1804004. https://doi.org/10.1002/adma.201804004.

(16) Liao, J.; Vallobra, P.; O’Brien, L.; Atxitia, U.; Raposo, V.; Petit, D.; Vemulkar, T.; Malinowski, G.; Hehn, M.; Martínez, E.; Mangin, S.; Cowburn, R. P. Controlling All-Optical Helicity-Dependent Switching in Engineered Rare-Earth Free Synthetic Ferrimagnets. Adv. Sci. 2019, 6 (24), 1901876. https://doi.org/10.1002/advs.201901876.

(17) Kichin, G.; Hehn, M.; Gorchon, J.; Malinowski, G.; Hohlfeld, J.; Mangin, S. From Multiple- to Single-Pulse All-Optical Helicity-Dependent Switching in Ferromagnetic Co / Pt $\begin{array}{lllllll}\text { Multilayers. } & \text { Phys. } & \text { Rev. Applied } & \text { 2019, } & 12 & \text { (2), } & 024019 .\end{array}$ https://doi.org/10.1103/PhysRevApplied.12.024019.

(18) Wilson, R. B.; Gorchon, J.; Yang, Y.; Lambert, C.-H.; Salahuddin, S.; Bokor, J. Ultrafast Magnetic Switching of GdFeCo with Electronic Heat Currents. Phys. Rev. B 2017, 95 (18), 180409. https://doi.org/10.1103/PhysRevB.95.180409.

(19) Xu, Y.; Deb, M.; Malinowski, G.; Hehn, M.; Zhao, W.; Mangin, S. Ultrafast Magnetization Manipulation Using Single Femtosecond Light and Hot-Electron Pulses. Adv. Mater. 2017, 29 (42), 1703474. https://doi.org/10.1002/adma.201703474.

(20) Avilés-Félix, L.; Olivier, A.; Li, G.; Davies, C. S.; Álvaro-Gómez, L.; Rubio-Roy, M.; Auffret, S.; Kirilyuk, A.; Kimel, A. V.; Rasing, Th.; Buda-Prejbeanu, L. D.; Sousa, R. C.; Dieny, B.; Prejbeanu, I. L. Single-Shot All-Optical Switching of Magnetization in Tb/Co MultilayerBased Electrodes. Sci. Rep. 2020, 10 (1), 5211. https://doi.org/10.1038/s41598-020-62104-w.

(21) Banerjee, C.; Teichert, N.; Siewierska, K. E.; Gercsi, Z.; Atcheson, G. Y. P.; Stamenov, P.; Rode, K.; Coey, J. M. D.; Besbas, J. Single Pulse All-Optical Toggle Switching of Magnetization without Gadolinium in the Ferrimagnet Mn2RuxGa. Nat. Commun. 2020, 11 (1), 4444. https://doi.org/10.1038/s41467-020-18340-9.

(22) Hashimoto, S. Adding Elements to the Co Layer in Co/Pt Multilayers. J. Appl. Phys. 1994, 75 (1), 438-441. https://doi.org/10.1063/1.355870.

(23) Ostler, T. A.; Evans, R. F. L.; Chantrell, R. W.; Atxitia, U.; Chubykalo-Fesenko, O.; Radu, I.; Abrudan, R.; Radu, F.; Tsukamoto, A.; Itoh, A.; Kirilyuk, A.; Rasing, T.; Kimel, A. Crystallographically Amorphous Ferrimagnetic Alloys: Comparing a Localized Atomistic Spin 
Model with Experiments. Phys. Rev. B 2011, $84 \quad$ (2), 024407. https://doi.org/10.1103/PhysRevB.84.024407.

(24) Gorchon, J.; Wilson, R. B.; Yang, Y.; Pattabi, A.; Chen, J. Y.; He, L.; Wang, J. P.; Li, M.; Bokor, J. Role of Electron and Phonon Temperatures in the Helicity-Independent All-Optical Switching of GdFeCo. Phys. Rev. B 2016, $94 \quad$ (18), 184406. https://doi.org/10.1103/PhysRevB.94.184406.

(25) Choi, G.-M.; Min, B.-C. Laser-Driven Spin Generation in the Conduction Bands of Ferrimagnetic Metals. Phys. Rev. B 2018, $97 \quad$ (1), 014410. https://doi.org/10.1103/PhysRevB.97.014410.

(26) Bergeard, N.; Hehn, M.; Mangin, S.; Lengaigne, G.; Montaigne, F.; Lalieu, M. L. M.; Koopmans, B.; Malinowski, G. Hot-Electron-Induced Ultrafast Demagnetization in Co / Pt Multilayers. Phys. Rev. Lett. 2016, $117 \quad$ (14), 147203. https://doi.org/10.1103/PhysRevLett.117.147203.

(27) Gottwald, M.; Hehn, M.; Lacour, D.; Hauet, T.; Montaigne, F.; Mangin, S.; Fischer, P.; Im, M.-Y.; Berger, A. Asymmetric Magnetization Reversal in Dipolarly Coupled Spin Valve Structures with Perpendicular Magnetic Anisotropy. Phys. Rev. B 2012, 85 (6), 064403. https://doi.org/10.1103/PhysRevB.85.064403.

(28) Beens, M.; Lalieu, M. L. M.; Deenen, A. J. M.; Duine, R. A.; Koopmans, B. Comparing All-Optical Switching in Synthetic-Ferrimagnetic Multilayers and Alloys. Phys. Rev. B 2019, 100 (22), 220409. https://doi.org/10.1103/PhysRevB.100.220409.

(29) Steil, D.; Alebrand, S.; Hassdenteufel, A.; Cinchetti, M.; Aeschlimann, M. All-Optical Magnetization Recording by Tailoring Optical Excitation Parameters. Phys. Rev. B 2011, 84 (22), 224408. https://doi.org/10.1103/PhysRevB.84.224408.

(30) Beens, M.; Duine, R. A.; Koopmans, B. S - d Model for Local and Nonlocal Spin Dynamics in Laser-Excited Magnetic Heterostructures. Phys. Rev. B 2020, 102 (5), 054442. https://doi.org/10.1103/PhysRevB.102.054442.

(31) Koopmans, B.; Malinowski, G.; Dalla Longa, F.; Steiauf, D.; Fähnle, M.; Roth, T.; Cinchetti, M.; Aeschlimann, M. Explaining the Paradoxical Diversity of Ultrafast Laser-Induced Demagnetization. Nature Mater. 2010, 9 (3), 259-265. https://doi.org/10.1038/nmat2593.

(32) Kittel, C. Introduction to Solid State Physics. Wiley Wiley, New York, ed 2004. 